\title{
Stepwise Treatment of Uterine Arteriovenous Malformation Complicated by a Giant Aneurysm: A Case Report
}

\author{
Vladislav V. Garbuzov ${ }^{1^{*}}$; Ravil F. Khafizov; Dmitry G. Gromov, $\mathrm{PhD}^{1}$; \\ Ekaterina V. Kozlova, $\mathrm{PhD}^{2}$ \\ 'Pirogov Russian National Research Medical University (RNRMU) \\ ${ }^{2}$ National Medical Research Center of Cardiology \\ Moscow, the Russian Federation
}

\begin{abstract}
Uterine arteriovenous malformation (AVM) is a rare condition that threatens severe uterine bleeding. We present a patient with this pathology, who was hospitalized twice within 5 months in serious condition, because she refused a hysterectomy. Ultrasonography of the uterus detected a vascular formation, the nature of which could be identified only on computed tomography (CT) angiography. Afferent vessels were embolized twice, and after the condition was stabilized, we performed a hysterectomy. For a long time, there was no recurrence of bleeding. The foregoing allows us to recommend the described tactics for the diagnosis and treatment of uterine AVM in women in similar clinical situations. (International Journal of Biomedicine. 2020;10(4):472-474.)
\end{abstract}

Key Words: arteriovenous malformation $\bullet$ embolization $\bullet$ aneurysm $\bullet$ vaginal bleeding

\section{Introduction}

Uterine arteriovenous malformation (AVM) is a very rare pathology, but potentially a life-threatening condition. ${ }^{(1)}$ Usually, uterine AVM manifests by periodic profuse uterine bleedings, pelvic pain, and anemia. ${ }^{(2)}$ Uterine AVMs are classified into congenital and acquired anomalies. Acquired anomalies are more common and usually follows a history of previous uterine trauma, such as curettage procedures, caesarean section, traumatic delivery, pregnancy termination, manual placenta removal, forceps delivery, vacuum extraction, or pelvic surgery. ${ }^{(4-6)}$ The acquired uterine AVMs often demonstrate complex and numerous arteriovenous communications, which may be due to the rich vascular network within the uterus. The initial imaging modality of choice for AVM is sonography with color and spectral Doppler analysis. ${ }^{(7)}$ The gold standard for diagnosis of uterine AVMs is digital subtraction angiography. ${ }^{(8)}$

\section{Case Presentation}

A 37-year-old woman in serious condition was admitted to the hospital with complaints of fatigue and copious spotting. Anamnesis showed that she had spotting for three years, with increased frequency and intensity during the last year, after separate diagnostic curettage. Hemoglobin level at admission was $58 \mathrm{~g} / \mathrm{L}$, the platelet count was $101 \times 10^{3} / \mu \mathrm{L}$. The patient underwent a blood transfusion, after which the hemoglobin level increased to $83 \mathrm{~g} / \mathrm{L}$. Ultrasound imaging showed enlargement of the uterus to $140 \mathrm{~mm} \times 107 \mathrm{~mm}$ and vascular mass in the myometrium closer to the left side wall with a diameter of 95 $\mathrm{mm}$. In the digital duplex mapping mode, signs of turbulent blood flow with possible thrombotic masses along the periphery were detected (Fig.1 A,B).

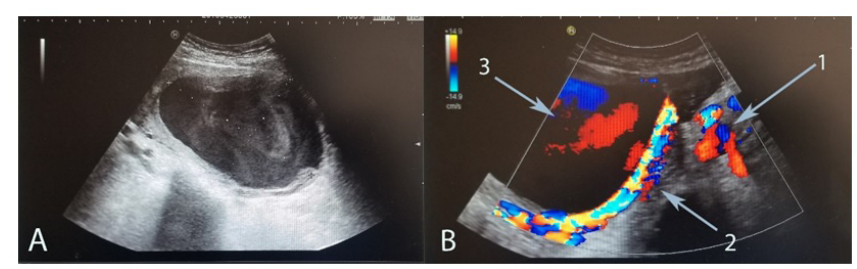

Fig. 1. (A) Ultrasound image shows vascular formation with a diameter of $95 \mathrm{~mm}$ located in the myometrium. (B) Color Doppler: 1. Dilation of the afferent and efferent vessels; 2. Parietal rapid blood flow from a large afferent; 3. Turbulent blood flow in the aneurysm cavity. 
The preliminary diagnosis was posttraumatic pseudoaneurysm after the traumatic diagnostic curettage. To clarify the diagnosis, CT angiography was performed. It revealed a vascular formation at the level of the iliac vessels on the left - a partially thrombosed aneurysm of a small vessel, arteriovenous malformation.

Despite the young age, the patient was offered a hysterectomy. Due to a serious health condition, in order to stop bleeding and prepare for surgery, the initial embolization of uterine arteries was performed. Selective angiography revealed dilated, convoluted vessels (the left uterine and the right uterine), with a contrast discharge from the distal segments into the cavity of the false aneurysm (Fig.2 A, B, C). During full contrasting, numerous collaterals between the the right uterine and left uterine were visualized. Further, these arteries with spirals were embolized. On the control angiogram, blood flow in these vessels was reduced, and contrast discharge into the aneurysm was not observed. The postoperative period was normal, uterine bleeding was stopped. The patient was discharged in satisfactory condition and was transferred to another hospital to have a hysterectomy; however, a hysterectomy was not performed.

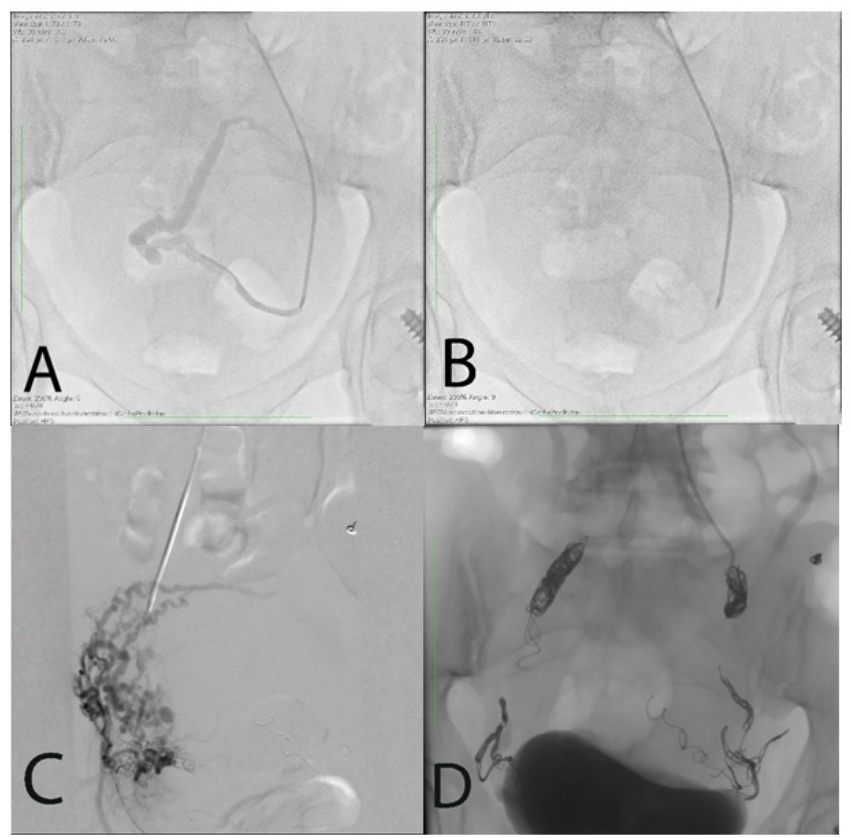

Fig.2. (A) Selective angiography: A large afferent branch of vascular malformation; (B) Selective angiography: the contrast agent enters the aneurysm; (C) The expanded convoluted right uterine artery with numerous collaterals in the area of the left uterine artery and the discharge of contrast from the distal segments into the cavity of the false aneurysm; (D) Control angiography: The embolization of the left uterine artery, right uterine artery, and left ovarian artery.

After 5 months, the patient was again hospitalized in a severe condition with a recurrence of uterine bleeding, weakness, dizziness, and tinnitus. The heart rate was 120 bpm, blood pressure - 100/60 mmHg. Blood hemoglobin was $36 \mathrm{~g} / \mathrm{L}$; platelet count was reduced to $76 \times 10^{3} / \mu$ l. After hemotransfusion, the hemoglobin level increased to $89 \mathrm{~g} / \mathrm{L}$. Duplex scanning revealed an aneurysm measuring $90 \mathrm{~mm} \times 87$ $\mathrm{mm} \times 80 \mathrm{~mm}$ with parietal thrombotic masses in the left wall of the uterus with a visible blood flow. In the region of the left internal iliac artery, there were multiple arterial branches with the main type of blood flow and venous collaterals. A CT scan of the small pelvis with contrast demonstrated the formation of a spindle-shaped form $42 \mathrm{~mm} \times 28 \mathrm{~mm}$ in size with lacunar filling with a contrast agent in the arterial phase on the left between the internal and external iliac arteries - a partially thrombosed aneurysm of a small vessel, arteriovenous malformation.

The patient underwent an intervention to stop bleeding. On selective angiograms of the right and left iliac arteries, hypertrophic afferent branches of malformation from obstructive, cystic, and internal genital arteries were contrasted. All afferent vessels with spirals for vascular embolization were embolized, with the additional injection of a crushed hemostatic sponge. Additionally, we performed bilateral occlusion of the internal iliac arteries with a tight arrangement of vessels with spirals for embolization (Fig.2 D). On control angiography, blood flow through the vessels was reduced; malformation was not contrasted.

The postoperative period was normal. The patient was transferred to a specialized department to have a hysterectomy. After 10 days, a hysterectomy was successfully performed. After another week, the patient was discharged in satisfactory condition. The vaginal discharge did not recur.

\section{Discussion}

Traditionally, uterine AVM has been diagnosed after hysterectomy using the histopathological examination. In this case, due to the morphological features of the pathology, only a computed tomography of the small pelvis with contrast allowed us to establish the correct diagnosis. It should be noted that selective digital subtraction angiography, which in such cases should be considered as the gold diagnostic standard, gives a complete picture of the formation angioarchitectonics. ${ }^{(8,9)}$ Due to the patient's initial serious condition, a hysterectomy was associated with an extremely high risk of death. In our opinion, the preliminary use of X-ray endovascular techniques, including bilateral internal iiac artery embolization, made it possible to stop bleeding rapidly, minimize intraoperative blood loss, and avoid fatal complications. Thus, the foregoing allows us to recommend the described tactics for the diagnosis and treatment of uterine AVM in women in similar clinical situations.

\section{Competing Interests}

The authors declare that they have no competing interests.

*Corresponding author: Vladislav V. Garbuzov, Pirogov Russian National Research Medical University (RNRMU), Moscow, Russia.E-mail: crimson.dawn105@gmail.com 


\section{References}

1. Cura M, Martinez N, Cura A, Dalsaso TJ, Elmerhi F. Arteriovenous malformations of the uterus. Acta Radiol. 2009 Sep;50(7):823-9. doi: 10.1080/02841850903008792..

2. Brown JV 3rd, Asrat T, Epstein HD, Oglevie S, Goldstein $\mathrm{BH}$. Contemporary diagnosis and management of a uterine arteriovenous malformation. Obstet Gynecol. 2008 Aug; 112(2 Pt 2):467-70. doi: 10.1097/AOG.0b013e3181719f7d.

3. Burrows PE. Vascular malformations involving the female pelvis. Semin Intervent Radiol. 2008 Dec;25(4):347-60. doi: 10.1055/s-0028-1102993.

4. Kuwata T, Matsubara S, Kaneko Y, Izumi A, Nakata M, Suzuki M. Asymptomatic uterine artery pseudoaneurysm after cesarean section. J Obstet Gynaecol Res. 2010 Apr;36(2):40510. doi: 10.1111/j.1447-0756.2009.01134.x.

5. Nagayama C, Gibo M, Nitta H, Uezato T, Hirakawa M, Masamoto H, Sakumoto K, Aoki Y. Rupture of pseudoaneurysm after vaginal delivery successfully treated by selective arterial embolization. Arch Gynecol Obstet. 2011 Jan;283(1):37-40. doi: 10.1007/s00404-009-1271-1.

6. Kim YA, Han YH, Jun KC, Jeon MK, Lee ES. Uterine artery pseudoaneurysm manifesting delayed postabortal bleeding. Fertil Steril. 2008 Sep;90(3):849.e11-4. doi: 10.1016/j.fertnstert.2007.07.1372.

7. Timmerman D, Wauters J, Van Calenbergh S, Van Schoubroeck D, Maleux G, Van Den Bosch T, Spitz B. Color Doppler imaging is a valuable tool for the diagnosis and management of uterine vascular malformations. Ultrasound Obstet Gynecol. 2003 Jun;21(6):570-7. doi: 10.1002/ uog. 159 .

8. Grivell RM, Reid KM, Mellor A. Uterine arteriovenous malformations: a review of the current literature. Obstet Gynecol Surv. 2005 Nov;60(11):761-7. doi: 10.1097/01. ogx.0000183684.67656.ba.

9. Müngen E. Vascular abnormalities of the uterus: have we recently over-diagnosed them? Ultrasound Obstet Gynecol. 2003 Jun;21(6):529-31. doi: 10.1002/uog.163. 